\title{
PEAK LOAD IN RAPID COMPRESSION TESTS : EXPERIMENTS AND MICROMECHANICAL MODELLING
}

\author{
Véronique Favier $^{1^{*}}$ and Helen Atkinson ${ }^{2}$ \\ ${ }^{1}$ Arts et Métiers ParisTech, LIM UMR CNRS 8006, ENSAM, 151 Bd de l’Hôpital, 75013 Paris, \\ France \\ ${ }^{2}$ Department of Engineering, University of Leicester, University Road, Leicester LE1 7RH, United \\ Kingdom
}

\begin{abstract}
Rapid compression tests are useful to characterize the semisolid behaviour in thixoforming conditions. The load-displacement curves typically display a peak load. The origin, the maximum value and the height of this peak are discussed comparing calculated results obtained using a micro-macro model and experimental data obtained in earlier work. As suggested by experiments, the calculated peak load originates from a 3D continuous solid skeleton, which breaks down under load. For the same initial semisolid structure, the model predicts that decreasing the ram speed decreases the maximum of the peak because of a strain rate effect. In addition, the peak load is found to be quite sensitive to initial internal structure, namely the volume fraction of entrapped liquid, which may change for various soaking times. Finally, the time for the solid skeleton breakdown is found to be in good agreement with experimental results.
\end{abstract}

KEYWORDS: Semisolids, Compression tests, Microstructure, Transient behaviour, Micromechanics

\section{INTRODUCTION}

Semisolid processing is a method of forming complexshaped components in the semisolid state. The processing is called thixoforming when the material gives a non-dendritic microstructure on reheating into the semisolid state. In that case, the solid fraction is usually higher than 0.5 . The processing is labelled as rheocasting when a liquid alloy is cooled into the semisemisolid state and gives a non-dendritic microstructure In that case, the solid fraction is usually lower than 0.5 . Rapid compression tests are useful to estimate the semisolid behaviour in thixoforming [1]. Indeed, the rate of compression is of the same order as thatn in industrial process. The solid fraction involved is higher than 0.5 . In addition, rapid compression tests provide the semisolid response in the transient regime. Modelling the transient behaviour is one of the main challenges in order to perform numerical simulations of optimised thixoforming. Various constitutive equations are proposed in literature (see e.g. [2]). The thixotropic behaviour is usually accounted for by means of a structure parameter, often designated by $\lambda$. The time derivative of $\lambda$ introduces the rate of buildup (agglomeration) of the solid particles and the rate of breakdown (desagglomeration). Recently, Favier et al [3] propose a constitutive equation which clarifies the mechanical role of liquid and solid and of their spatial distribution within the material on the overall behaviour of semisolids. To do so, they use micromechanics and homogenization techniques. This modelling successfully described the steady-state behaviour and was extended to non steady-state (transient) behaviour [4]. The present objective is essentially to correlate the mechanical response of semisolid slug, under compression loadings, to the deformation mechanisms and the microstructure of the material. Section 2 deals with the basic concepts of the modelling. Section 3 focuses on the semisolid slug response under compression loadings. The results, concerning both the influence of ram speed and initial material microstructure, are compared qualitatively to the experimental results of Liu et al [5].

\section{MICROMECHANICAL MODELLING}

The proposed micro-macro modelling is based on the following statements (further details are given in [3]):

In semisolids having a solid fraction $f^{s}$, four mechanical "phases" within the material are identified: the solid globules, the solid bonds between the solid globules, the free liquid and the

\footnotetext{
* Corresponding author: LIM, ENSAM, 151 Bd de l'Hôpital, 75013 Paris, France, +33 608695444, +33 144246382, veronique.favier@paris.ensam.fr
} 
liquid entrapped within the solid globules. These phases do not play a similar mechanical role: the deformation mainly takes place in local sites such as the bonds between the solid grains and the liquid that is not entrapped in the agglomerated solid particles. From a statistical point of view, the material is represented via a coated inclusion: the inclusion is composed of both solid and liquid with volume fractions $f_{I}^{S}$ and $f_{I}^{l}$ respectively, to represent entrapped liquid within solid particles; the coating (the active zone which carries most of the deformation) consists of the solid bonds and the non-entrapped liquid with volume fractions $f_{A}^{S}$ and $f_{A}^{l}$ respectively.

The volume fraction of the entrapped liquid is given by:

$$
f_{\text {entrapped liquid }}=f_{I}^{l} \times f_{I}
$$

$\checkmark \quad$ The liquid phase is regarded as a Newtonian fluid (Equation 2) whereas the solid phase is considered as viscoplastic (Equation 3).

$$
S_{i j}^{l}=2 \mu^{l} \dot{\varepsilon}_{i j}^{l} \text { with } \mu^{l}=K^{l}
$$

for the Newtonian liquid (suffix $l$ ) and

$$
S_{i j}^{g, b}=2 \mu^{g, b} \dot{\varepsilon}_{i j}^{g, b} \text { with } \mu^{g, b}=K^{g, b}\left(\frac{\sqrt{3} \dot{\varepsilon}_{e q}^{g, b}}{\dot{\gamma}_{0}^{g, b}}\right)^{m^{g, b}-1}
$$

for the viscoplastic solid globules (suffix $g$ ) and the solid bonds (suffix $b$ ).

$S$ and $\dot{\varepsilon}$ are the deviatoric stress and strain rate tensors, respectively. $\mu$ is the viscosity. $\dot{\varepsilon}_{e q}$ is the von Mises equivalent strain rate. $K$ is the consistency. $m$ is the power-law index (strain rate sensitivity parameter). $\dot{\gamma}_{0}$ is the reference strain rate taken equal to $1 \mathrm{~s}^{-1}$.

$\checkmark$ A self-consistent approximation is used at two scales to determine the semisolid viscosity $\mu_{S S}$. First, the viscosity of the inclusion $\mu_{I}$ and of the active zone $\mu_{A}$, associated with the respective volume fractions $f_{I}$ and $f_{A}$, are calculated from the liquid and solid behaviour using a classical selfconsistent scheme. Then the viscosity of the semisolid is deduced from the inclusion and the active zone viscosities using the self-consistent estimation applied to the specific morphology of the coated inclusion.
The evolution of the microstructure as a function of the strain rate is captured through an internal variable describing the degree of agglomeration of the solid skeleton. This internal variable is the solid fraction of the active zone $f_{A}^{s}$ measuring the amount of bonds. The evolution law introduces only disagglomeration owing to shear inside the bonds (Equation 4) [4]. This choice is justified by the fact that in rapid compression tests, desagglomeration process dominates with regard to the agglomeration process [2].

$$
\dot{f}_{s}^{A}=-\left(1-f_{s}\right) \frac{\dot{\gamma}_{\text {bonds }}}{\gamma_{c}} f_{s}^{A}
$$

The shear rate within the solid bonds $\dot{\gamma}_{\text {bonds }}$ is naturally given by the micro-macro modelling and is equal to:

$$
\dot{\gamma}_{\text {bonds }}=\sqrt{3}\left(\dot{\varepsilon}_{A}^{s}\right)_{e q}
$$

In addition, we postulate that the solid bonds break as soon as the local shear reaches a critical value $\gamma_{c}$.

\section{RESULTS AND DISCUSSION}

\subsection{SIMULATIONS OF COMPRESSION TESTS}

We simulated compression tests using the micro-macro model assuming uniform stress fields. We consider solid fraction higher than 0.5 to be in agreement with usual compression tests. Since the solid fraction is quite high and the fact that the material may be at rest prior to the application of the load, we assume that there is a continuous solid skeleton initially within the material. More precisely, we followed the test parameters of [5]. The ram speed used was $500 \mathrm{~mm} / \mathrm{s}$, unless otherwise stated. The height and the diameter of the initial slug are $42 \mathrm{~mm}$ and $36 \mathrm{~mm}$, respectively. Fig. 1 displays the evolution of the overall equivalent shear rate vs the specimen displacement throughout the test. The overall equivalent shear rate increased from 20 to $180 \mathrm{~s}^{-1}$. This range and the shear rate evolution during the test may be different from the experimental since we consider pure uniaxial compression in the modelling whereas we do not have pure uniaxial compression in experiments.

The solid fraction associated with the experiments described in [5] is not indicated. As a first approach, we consider that the solid fraction is 0.7 . Model parameters were chosen to be qualitatively consistent with experimental results of [5]. Parameters associated with the material representation are listed in Table 1, unless otherwise stated. Rheological parameters are listed in Table 2 and remain constant for all the subsequent simulations. 


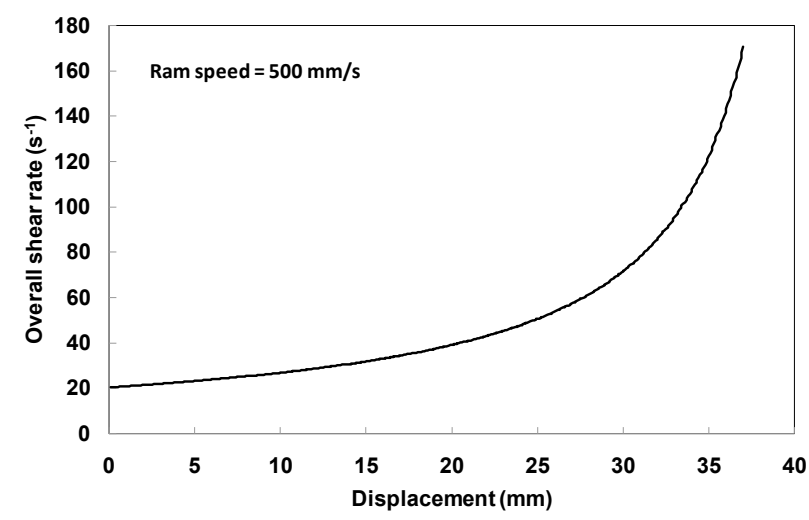

Figure 1: Modelled evolution of the overall equivalent shear rate throughout compression test at a ram speed of $500 \mathrm{~mm} / \mathrm{s}$.

Table 1: Parameters associated with the representation of the material for $f_{\text {entrapped liquid }}=29,5 \%$

\begin{tabular}{llll}
\hline$f_{\text {Ainitial }}^{s}$ & $\gamma_{c}$ & $f_{A}$ & $f_{c}$ \\
\hline 0,5 & 1.0 & 0,01 & 0,4 \\
\hline
\end{tabular}

Table 2: Rheological parameters

\begin{tabular}{|c|c|c|c|}
\hline & Solid & & Liquid \\
\hline$K^{g}$ & $K^{b}$ & $m^{g}=m$ & $K^{l}$ \\
\hline $8 \times 10^{5} \mathrm{~Pa}$ & $6 \times 10^{5}$ Pa.s & 0.3 & $1.8 \times 10^{-1} \mathrm{~Pa} . \mathrm{s}$ \\
\hline
\end{tabular}

\subsection{RESULTS OF COMPRESSION TESTS}

\subsubsection{Load-displacement curve}

Figure 2 shows the load-displacement curves for compression tests at $250 \mathrm{~mm} / \mathrm{s}, 500 \mathrm{~mm} / \mathrm{s}$ and 1000 $\mathrm{mm} / \mathrm{s}$. The load typically increases up to a maximum and thereafter strongly decreases and increases again as often experimentally observed (see e.g. [2,5]). From the modelling point of view, we found that the presence of the peak is related to the breakdown of the continuous 3D solid skeleton. When the critical shear is reached, the bonds break. The solid skeleton is thus progressively broken up and entrapped liquid is released. After the peak load, the material becomes a suspension with solid agglomerates. At this step, the strain rate and the strain are mostly carried by the free liquid. Consequently, the bonds are not broken anymore and the structure of the material remains the same up to the end of the test. During this last stage, the strong increase of the load is due to the high increase of strain rate as showed in Figure 1.

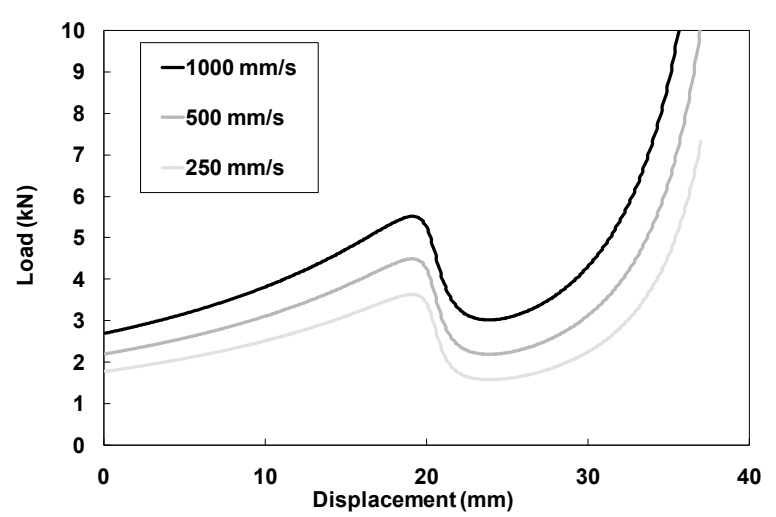

Figure 2: Load-displacement curves for various ram speeds of $1000 \mathrm{~mm} / \mathrm{s}, 500 \mathrm{~mm} / \mathrm{s}$ and $250 \mathrm{~mm} / \mathrm{s}$.

Concerning the response before the peak, the model predicts an initial infinite slope: the load increases instantly when the test starts. The initial value of the load results from the viscoplastic description of the material behaviour and the instantaneous change of the overall shear rate from 0 to $20 \mathrm{~s}^{-1}$ (Figure 1). Then, the load slowly increases up to the peak. This first stage does not exist in the experiments: the load is found to strongly increase from zero to the maximum value [5]. These discrepancies between literature experimental and calculated results show clearly that the model does not capture properly the behaviour of semisolids just after the application of the load. We think that this is due to the pure viscoplastic description of the behaviour and mechanical interactions. In the following, we will focus on the predicted behaviour from the peak load up to the end of the test.

\subsubsection{Influence of the ram speed}

Decreasing ram speed leads to a decrease of the load throughout the test and more specifically a decrease of the level and the height of the peak load. The peak loads appear at the same displacement value. These results are in qualitative agreement with experimental results of [5]. The analysis of the calculated results shows that the solid fraction in the active zone, $f_{A}^{s}$, is the same up to the peak load whatever the ram speed. As a result, the decrease of the peak load due to the decrease of ram speed does not come from microstructural changes as suggested by Liu et al [5] but is related to the strain rate sensitivity of the solid skeleton behaviour. When the ram speed is lower, the strain rate inside the solid bonds is also lower, and hence, more time is needed to reach the critical shear to break the bonds. Similarly, more time is needed to reach the displacement at the peak. Consequently, the solid fraction in the active zone and the disagglomeration process are directly related to the overall displacement but not to the overall strain rate. The overall strain rate controls the height of the peak and the time to reach the displacement required to break down the solid skeleton. 


\subsubsection{Influence of the initial internal structure of semisolid}

Figure 3 displays the load-displacement curves for various initial entrapped liquid volume fractions. These values mimic the effect of the soaking time. When the soaking time increases, the microstructure changes from a dendritic-type morphology to a more spheroidal particle morphology [5]. Consequently, some liquid entrapped initially is released during the soaking time. In other words, we consider that the entrapped liquid volume fraction decreases when the soaking time increases. The entrapped liquid volume fraction is calculated using Equation (1) and is indicated in Figure 3 . Note that the values of entrapped liquid amount are found to be much higher than those experimentally determined using 3D X-Ray tomography and image analysis after quenching [6]. Indeed, most of the liquid is considered entrapped inside the globules while Terzi et al [6] estimate that around $10 \%$ of solidified liquid is entrapped. This discrepancy may be explained by the fact that the cooling rate of quenching is too low to freeze the microstructure, and hence, the real liquid fraction is underestimated [6]. More probably, it comes from the strong contrast between the solid and liquid consistency $\left(10^{7}\right)$ which intensifies the mechanical role of the active zone in the model. Indeed, very low volume fraction of active liquid (resp. active solid) is required to control the overall properties. Further investigations are required to clarify this point.

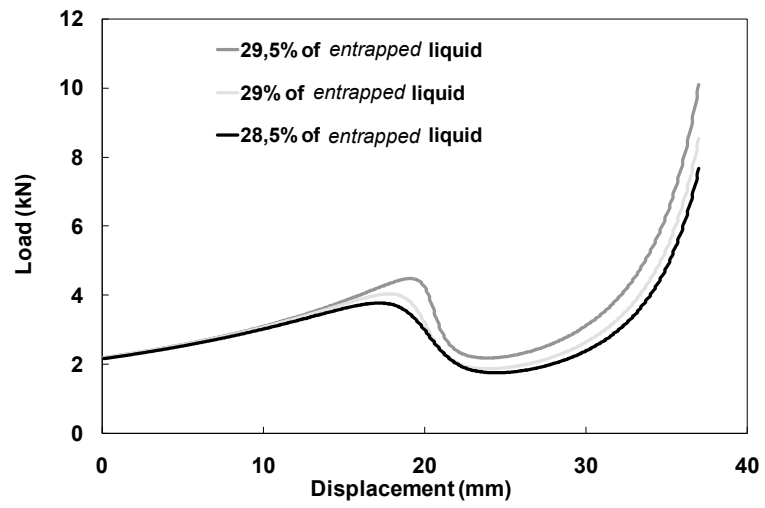

Figure 3: Load-displacement curves for various initial volume fractions of entrapped liquid and a ram speed of $500 \mathrm{~mm} / \mathrm{s}$.

Figure 3 shows that the peak load decreases when the entrapped liquid volume fraction is diminished. Indeed, reducing the entrapped liquid amount leads to having more liquid active during the flow and hence, lower loads. This result is consistent with the experimental results on the impact of the soaking times shown in [5]. It is worth noticing that the sensitivity of the response to the entrapped liquid volume fraction is high. The peak load is reduced by $16 \%$ when the entrapped liquid amount is decreased from $29.5 \%$ to $28.5 \%$. Following [5], we calculate the time taken to destroy the continuous skeleton (Table 3). To do so, we estimate the displacement between the peak load and the minimum of the load and account for the ram speed. We obtain a solid skeleton breakdown around $10 \mathrm{~ms}$ which is in very good agreement with Liu et al's results [5].

Table 3: Time for solid breakdown for various initial volume fractions of entrapped liquid

\begin{tabular}{cccc}
\hline $\begin{array}{c}\text { Volume fraction of } \\
\text { entrapped liquid }\end{array}$ & $29,5 \%$ & $29 \%$ & $28,5 \%$ \\
\hline $\begin{array}{c}\text { Time for solid skeleton } \\
\text { breakdown }\end{array}$ & $9 \mathrm{~ms}$ & $12 \mathrm{~ms}$ & $14 \mathrm{~ms}$ \\
\hline
\end{tabular}

\section{CONCLUSION}

In this paper, the load-displacement curve for semisolids under compression tests is modelled and discussed. The material constitutive equation is given by a micro-macro model which relates the microstructure and the deformation mechanisms to the overall semisolid behaviour. The peak load observed in the loaddisplacement curves originates from a $3 \mathrm{D}$ continuous solid skeleton, which breaks down under load, in good agreement with literature. Decreasing the ram speed decreases the maximum and the height of the peak. The maximum value is clearly related to a strain rate effect. The height of the peak is related to the disagglomeration of the solid phase. In addition, we found that the peak load is sensitive to initial internal structure, namely the volume fraction of entrapped liquid. Less entrapped liquid, due to a longer soaking time for example, reduces the peak load. The time for solid skeleton breakdown is found to be in very good agreement with experimental results of Liu et al [5].

\section{ACKNOWLEDGEMENT}

The authors thank the Royal Society of United Kingdom and COST 541 project for their financial support.

\section{REFERENCES}

[1] Kapranos P., Liu, T.Y.: Atkinson H.V., Kirkwood D.H.: Investigation into the rapid compression of semi-solid alloy slugs. Journal of Materials Processing Technology. 111:31-36, 2001

[2] Atkinson H.V.: Modelling the semisolid processing of metallic alloys. Progress in Materials Science. 50:341412, 2005

[3] Favier V., Rouff C., Bigot R., Berveiller M., Robelet M: Micro-macro modeling of the isothermal steady-State behaviour of semi-Solids. International Journal of Forming Processes. 7:177-194, 2004

[4] Favier V., Manceau M.: Transient semi-solid behaviour: modelling step-change up and step-change down in shear rate using a micromechanical approach. In: $12^{\text {th }}$ International ESAFORM Conference on Material Forming, submitted, 2009

[5] Liu T.Y., Atkinson H.V., Kapranis P., Kirkwood D.H., Hogg S.C.: Rapid compression of aluminium alloys and its relationship to thixoformability. Metallurgical and Materials Transactions A. 34:1545-1554, 2003

[6] Terzi S., Limodon N., Boller E., Salco L., Suéry M.: XRay microtomography investigation of the effect of $\mathrm{Ba}$ additions on the microstructure of partially remelted Al- 
$4 \% \mathrm{Cu}$ alloys. Solid State Phenomena.141-143:475-480,

2008 